\title{
NOTES
}

\section{Fibers from Multi-walled Carbon Nanotube/Polyacrylonitrile Composites}

\author{
Biao WANG, ${ }^{\dagger}$ Huaping WANG, Bindun Hong, and Yumei ZHANG \\ State Key Laboratory for Modification of Chemical Fiber and Polymers, \\ College of Material Science and Engineering, Dong Hua University, Shanghai, 200051, China
}

(Received November 9, 2004; Accepted January 20, 2005; Published May 15, 2005)

KEY WORDS Carbon Nanotubes / Composite / Polyacrylonitrile Fibers / In-situ Polymerization / [DOI 10.1295/polymj.37.376]

Carbon nanotubes (CNT) exhibit excellent mechanical and electronic properties and hence have received much attention in recent years for wide potential applications such as field emitters, scanning probes, sensors, and nanoelectronics. ${ }^{1-6}$ One of the most intriguing applications of CNT is polymer/CNT nanocomposites in which the nanotube architecture is established with a host polymer matrix. ${ }^{7-17}$ The mechanical and novel electronic characteristics of CNT make them ideal candidate as fillers in lightweight polymer composites designed for structural or functional applications. In the past few years, a great quantity of research on fabrication of various polymer/CNT composites has been reported. Polymers such as epoxy, ${ }^{12,13}$ poly(methyl methacrylate) (PMMA), ${ }^{14}$ polycarbonate (PC) ${ }^{15}$ poly(ethylene oxide),${ }^{16}$ as well as poly(vinyl acetate), ${ }^{17}$ have been used as matrixes. However, development in polymer/CNT nanocomposites has been strongly limited by problems associated with the dispersion of the fillers and load transfer across the polymer-CNT interface. ${ }^{18}$ Reported methods are still microscale and not suitable for commercial composite manufacturing.

Polyacrolonitrile (PAN) co-polymers are commercially important and used for developing porous and activated carbon for a variety of applications. ${ }^{19,20} \mathrm{Re}-$ cently, CNT/PAN ultra-fine fibers have been fabricated by co-electrospinning. ${ }^{18}$ CNT/PAN films have been prepared for supercapacitor electrode application. ${ }^{21}$ CNT effectively disperses in PAN matrix. In this study, multi-walled carbon nanotube (MWNT)/ PAN composites were produced by in-situ polymerization. Fibers from these composites were prepared by traditional wet-spinning, which is simple and for large-scale production.

\section{EXPERIMENTAL}

Acrylonitrile monomer (AN), methyl methacrylate (MMA), 2,2'-azobisisobutyronitrile (AIBN) were supplied by Aldrich Chemicals. MWNTs were produced by a chemical vapor deposition method described in our previous work. ${ }^{22,23}$ SEM and TEM images in Figure 1 show typical length and diameter of MWNT as $4-6 \mu \mathrm{m}$ and $30-50 \mathrm{~nm}$, respectively. The asprepared MWNTs were purified with a mixture of concentrated nitric and sulphuric acids in a ratio of $1: 3$ followed by washing, filtrating and drying, ${ }^{24}$ and then treated with aqueous solution of $3.0 \mathrm{wt} \%$ Gum Arabic by the method proposed by Regev et al. ${ }^{25}$ Spinning dopes of MWNT/PAN composites were prepared by in-situ polymerization using AIBN as the initiator and $51 \mathrm{wt} \% \mathrm{NaSCN}$ solution as the solvent. ${ }^{26}$ AIBN quantity, reaction time, and temperature were controlled to have uniform molecular weights in all composites. Reactions to produce dopes of these composites were carried out with stirring in a flask containing AN monomer, MMA, AIBN and Gum Arabic-treated MWNT and $51 \mathrm{wt} \% \mathrm{NaSCN}$ solution. ${ }^{26}$ The mixture was stirred and heated at $78^{\circ} \mathrm{C}$ for $1 \mathrm{~h}$ and the spinning dope was obtained after 5 min demonomerizing. The $\mathrm{P}(\mathrm{AN}-\mathrm{co}-\mathrm{MMA})$ copolymer ratio is $\sim 90: 10$ (in feed) and polymer molecular weight is $\sim 60000 \mathrm{~g} \mathrm{~mol}^{-1} .{ }^{26} \mathrm{P}(\mathrm{AN}-c o-\mathrm{MMA})$ copolymer is referred to as PAN.

The prepared dopes were used to produce MWNT/ PAN composite fibers on a wet-spinning machine. The schematic of wet-spinning process is shown in Figure 2. A three-hole spinneret of $500 \mu \mathrm{m}$ diameter and a 400 mesh stainless steel filter pack were used in the spinning process. The dope temperature was maintained at $80^{\circ} \mathrm{C}$ and the take-up roller speed was

${ }^{\dagger}$ To whom correspondence should be addressed (E-mail: Wbiao2000@dhu.edu.cn). 
(a)

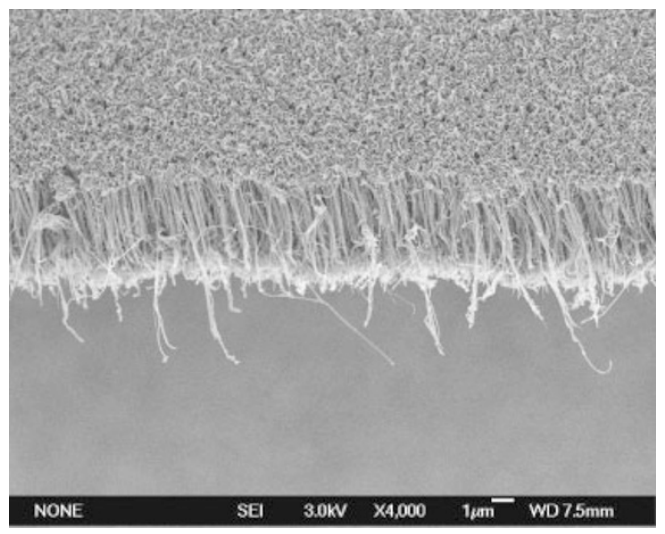

(b)

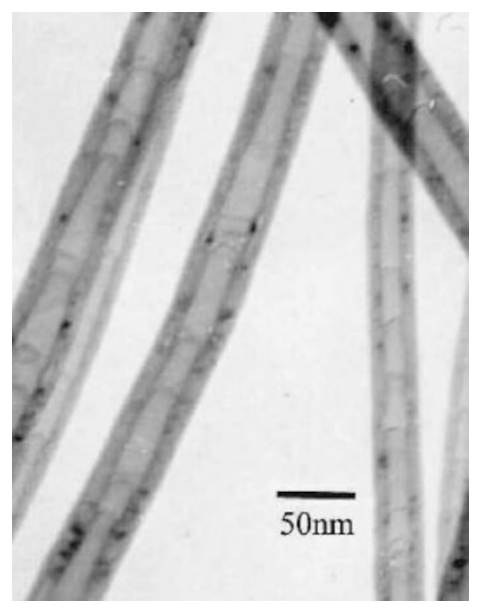

Figure 1. SEM and TEM images of MWNT: (a) A SEM image of aligned MWNT synthesized on silicon surface by a chemical vapor deposition method. (b) A TEM image of purified MWNT.
$10 \mathrm{~m} / \mathrm{min}$ to avoid jet stretch. NaSCN/water ratios for baths I, II, III were 10:90, 2:98 and 0:100, respectively. Bath I and II were maintained at $\sim 5^{\circ} \mathrm{C}$ and $\sim 55^{\circ} \mathrm{C}$, respectively, while bath III was maintained at $90^{\circ} \mathrm{C}$. No fiber drawing took place in baths I and II, while fibers were drawn in bath III and allowed to relax in subsequent drying, carried out on a hot plate at $120^{\circ} \mathrm{C}$. For structure and properties comparisons, all fibers were drawn to a draw ratio of 5.0.

MWNT and their composite fibers were examined by scanning electron microscopy (SEM; JSM-6300F and Hitachi S-530) and Transmission electron microscopy (TEM; JEOL 100CX2) to characterize morphology and uniformity. Fiber mechanical properties were determined using a dynamic mechanical analyzer system (PerkinElmer DMA7e). A frequency of $10 \mathrm{~Hz}$, a static force of $0.1 \mathrm{~N}$ and amplitude of $5.0 \mu \mathrm{m}$ were used and the temperature ramp was $5.0^{\circ} \mathrm{C} / \mathrm{min}$. Volume resistivity of the composite fiber was determined by measuring DC resistance along the longitudinal direction through fibers approximately $20 \mathrm{~mm}$ in length. An electrometer Model ZC25-4 was used to measure high resistance by the method of Potschke et al. ${ }^{15}$ According to ASTM D4496 and D257 in the American Society for Testing and Materials, volume resistivity $\left(\rho_{\mathrm{v}}\right)$ was calculated as:

$$
\rho_{\mathrm{v}}=\frac{\pi d^{2} n R_{\mathrm{v}}}{4 l}
$$

where $d$ and $l$ are diameter and length of the fibers, respectively, $n$ is the number of the measured fibers, and $R_{\mathrm{V}}$ is the measured resistance. The reported values represent means of 10 samples, where standard deviation relative to a mean was less than $15 \%$.

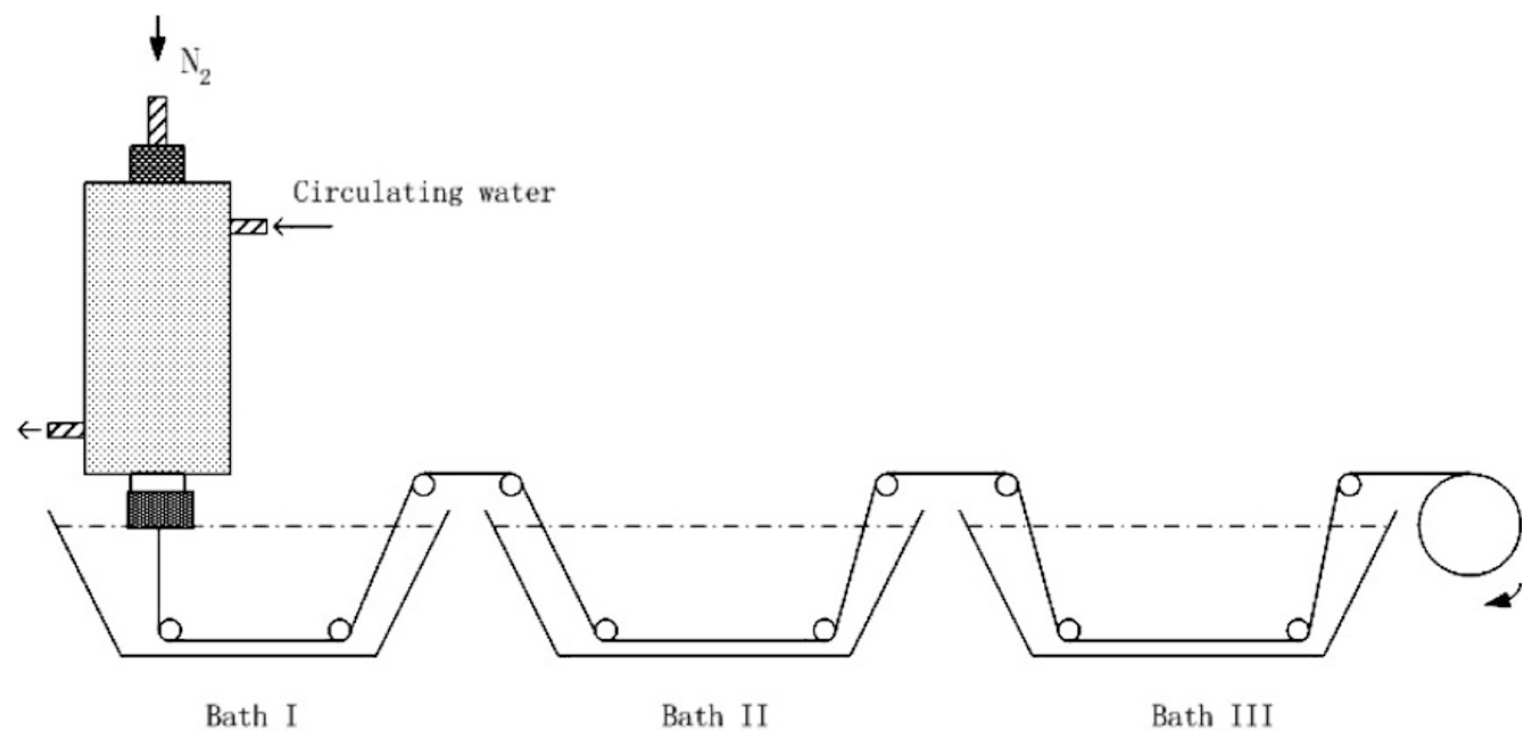

Figure 2. Schematic of wet-spinning. 
(a)

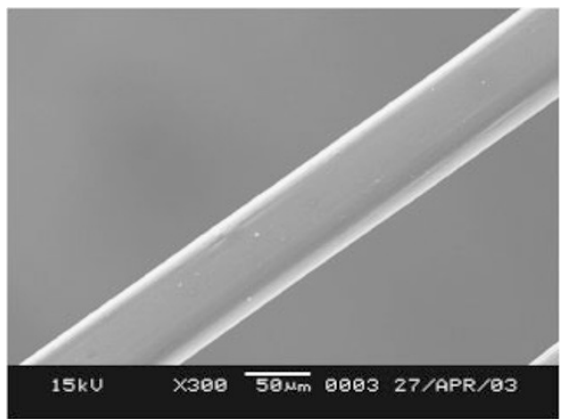

(b)

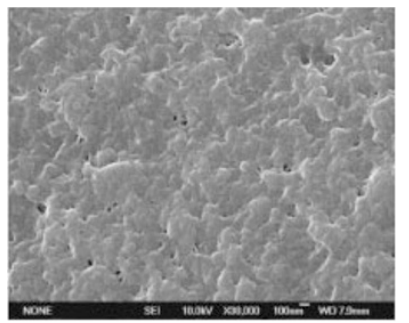

(C)

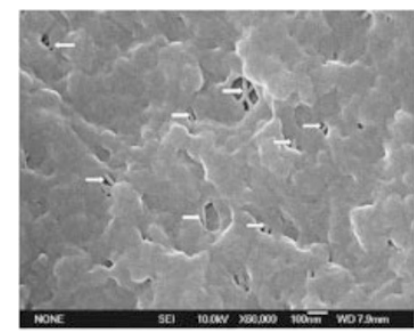

Figure 3. SEM images of composite fibers: (a) PAN fiber with $0.5 \mathrm{wt} \%$ MWNT loading; (b) a cross-sectional image of MWNT/PAN composite fiber ( $0.5 \mathrm{wt} \%$ MWNT loading); (c) higher magnification view of MWNT embedded in PAN matrix ( $0.5 \mathrm{wt} \%$ MWNT loading, arrows pointed to the MWNTs).

\section{RESULTS AND DISCUSSION}

Figure $1 \mathrm{~b}$ shows a TEM image of purified MWNT. From this figure, the surface of the nanotube is very clean and most of the impurity phases such as amorphous carbon and graphitic nanoparticles are removed. SEM images of a MWNT/PAN fiber, given in Figure 3, show that the surface of as-produced fiber is very smooth (Figure 3a) and MWNTs uniformly distributed and well dispersed in the matrix (Figures $3 \mathrm{~b}$ and $3 \mathrm{c}$ ). MWNTs are extremely hydrophobic and require a stabilizer to achieve a stable aqueous suspension. Here, Gum Arabic, an effective agent to disperse and separate MWNT in aqueous solutions, ${ }^{25}$ was used for this purpose. Physical adsorption of Gum Arabic on MWNT surface makes the MWNT well dispersed in $51 \mathrm{wt} \% \mathrm{NaSCN}$ aqueous solution, which results in uniformly dispersing and stabilizing MWNT in the dopes of MWNT/PAN during in-situ polymerization. Gum Arabic treatment and in-situ polymerization process are effective for the dispersion of MWNT and contribute to successful spinning.

The mechanical properties of as-prepared MWNT/ PAN fibers were investigated using a dynamic mechanical analyzer system. At each MWNT concentration, several samples were measured under the same conditions. Figure 4a shows the storage modulus $\left(E^{\prime}\right)$ of one set of samples. $30^{\circ} \mathrm{C}$ storage moduli $\left(E^{\prime} \mathrm{s}\right)$ from (a)

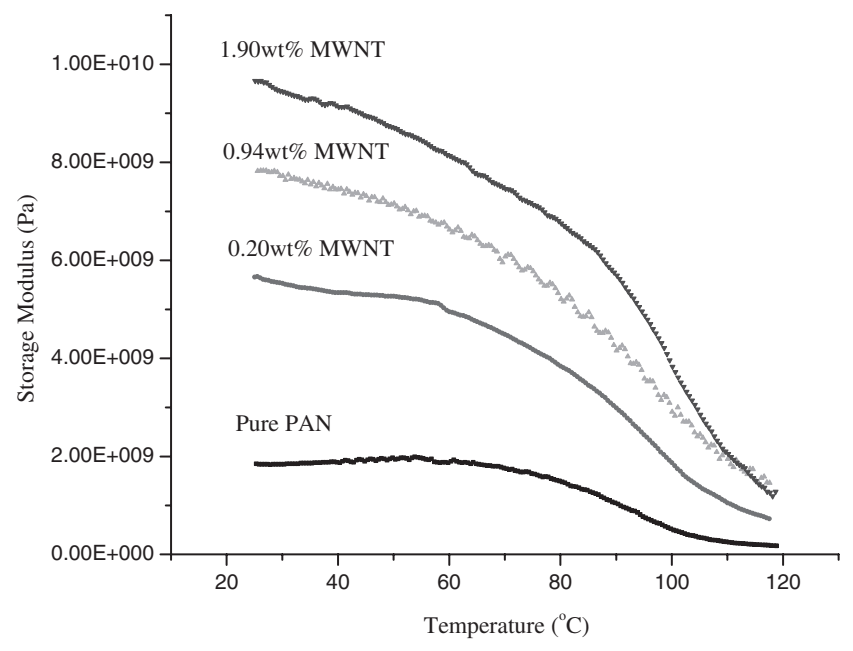

(b)

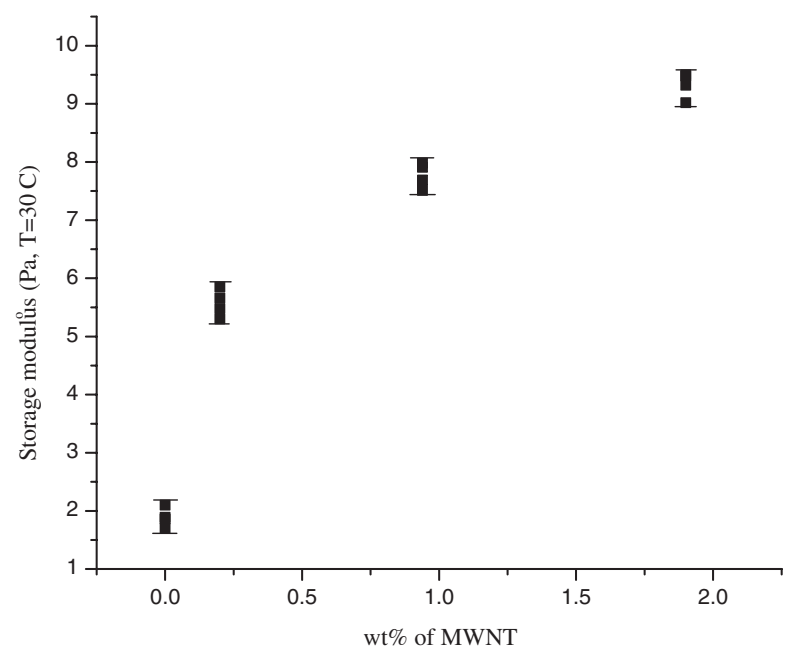

Figure 4. (a) Storage modulus as a function of temperature for pure PAN and MWNT/PAN composite fibers; (b) $30^{\circ} \mathrm{C}$ storage moduli $\left(E^{\prime} \mathrm{s}\right)$ from all samples measured (several samples with the same MWNT concentration).

all the samples measured are plotted in Figure $4 \mathrm{~b}$ as vs. wt $\%$ of MWNT loading. Storage moduli of MWNT/PAN fibers are significantly higher than that of the pure PAN in the entire temperature range of the experiment, and increase monotonically with MWNT loading. The averaged $30^{\circ} \mathrm{C} E^{\prime}$ (over several samples with the same MWNT concentration) increased from $1.89 \mathrm{GPa}$ for pure PAN fiber to 9.32 $\mathrm{GPa}$ for $1.9 \mathrm{wt} \% \mathrm{MWNT} / \mathrm{PAN}$ fiber. In contrast, when MWNTs were used without Gum Arabia treatment and/or the composites were prepared without using in-situ polymerization, wet spinning was not possible and the obtained fibers were asymmetrical with poor mechanical properties. However, when the temperature increased to $140^{\circ} \mathrm{C}$, there appeared no visible $\tan \delta$ (the ratio of the complex to real modulus) peak for MWNT/PAN fibers (data are not shown in 


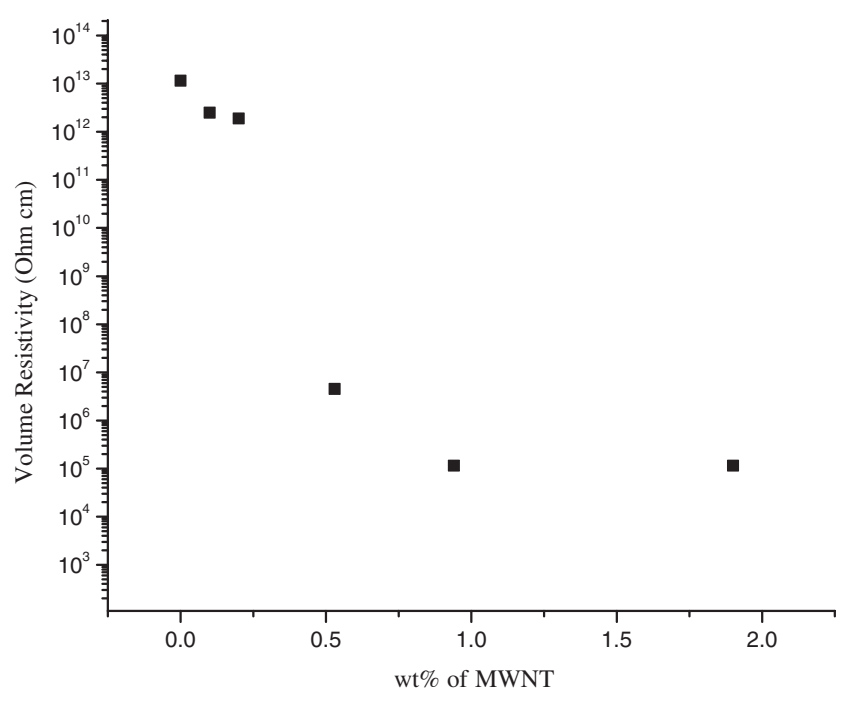

Figure 5. Effects of MWNT loading on volume resistivity.

this paper), while for pure PAN fibers, the $\tan \delta$ peak position was at $\sim 98^{\circ} \mathrm{C}$. The $\tan \delta$ peak thus broadens greatly towards higher temperature because of MWNT in PAN matrix. The motion of PAN molecules closer to MWNT would be more constrained than those farther away. ${ }^{27}$ Broadening of $\tan \delta$ peak may be attributed to interaction between PAN and MWNT; this is being studied at present.

Figure 5 shows the effect of adding MWNT on volume resistivity. At very low concentrations of MWNT, resistivity gradually decreased with nanotube content. At $0.5 \mathrm{wt} \%$, a significant reduction in resistivity at $10^{6} \Omega \mathrm{cm}$ was observed. This stepwise change in resistivity may result from the formation of an interconnected structure of MWNT and can be regarded as an electrical percolation threshold (EPT). Thus, at $\sim 0.5 \mathrm{wt} \%$ MWNT loading, a very high percentage of electrons flow through the sample due to an interconnecting conductive pathway. Compared to the carbon-black-based polymer composites,${ }^{28}$ MWNT/PAN composites show lower EPT. This reduction in EPT is due to the large aspect ratio of MWNT relative to carbon black, which requires fewer particle contacts to form a conductive network throughout the matrix.

Acknowledgment. This project was supported by Science and Technology Committee of Shanghai (0352nm064), China Petrochemical Corporation and Science and Technology development Foundation of Dong Hua University, China.

\section{REFERENCES}

1. S. Tan, A. Verschueren, and C. Dekker, Nature, 393, 49
(1998).

2. W. A. de Heer, J. M. Bonard, K. Fauth, A. Châtelain, L. Forro, and D. Ugarte, Adv. Mater., 9, 87 (1997).

3. Q. H. Wang, T. D. Corrigan, Y. J. Dai, R. P. H. Chang, and A. R. Krauss, Appl. Phys. Lett., 70, 3308 (1997).

4. A. N. Cleland and M. L. Roukes, Nature, 392, 160 (1998).

5. H. Dai, J. H. Hafner, A. G. Rinzler, D. T. Colbert, and R. E. Smalley, Nature, 384, 147 (1996).

6. W. B. Choi, D. S. Chung, J. H. Kang, H. Y. Kim, Y. W. Jin, I. T. Han, Y. H. Lee, J. E. Jung, N. S. Lee, G. S. Park, and J. M. Kim, Appl. Phys. Lett., 75, 3129 (1999).

7. L. Cai, H. Tabata, and T. Kawai, Appl. Phys. Lett., 77, 3105 (2000).

8. M. C. Hersam, A. C. F. Hoole, S. J. Shea, and M. E. Welland, Appl. Phys. Lett., 72, 915 (1998).

9. F. Ruiz, W. D. Sun, F. H. Pollak, and C. Venkatraman, Appl. Phys. Lett., 73, 1802 (1998).

10. G. B. M. Fiege, A. Altes, R. Heiderhoff, and L. J. Balk, J. Phys. D: Appl. Phys., 32, 13 (1999).

11. S. Gomes, N. Trannoy, and P. Grossel, Meas. Sci. Technol., 10, 805 (1999).

12. P. Ajayan, O. Astephan, C. Colliex, and D. Trauth, Science, 264, 1212 (1994).

13. X. Gong, J. Liu, S. Baskaran, R. Voise, and J. Young, Chem. Mater., 12, 1049 (2000).

14. Z. Jia, Z. Wang, C. Xu, J. Liang, B. Wei, D. Wu, and S. Zhu, Mater. Sci. Eng., A, 271, 395 (1999).

15. P. Potschke, T. D. Fornes, and D. R. Paul, Polymer, 43, 3247 (2002).

16. Z. X. Jin, X. Sun, G. Q. Xu, S. H. Goh, and W. Ji, Chem. Phys. Lett., 318, 505 (2000).

17. J. C. Grunlan, A. R. Mehrabi, M. V. Bannon, and J. L. Bahr, Adv. Mater., 16, 150 (2004).

18. F. Ko, Y. Gogotsi, A. Ali, N. Naguib, H. Ye, G. Yang, C. Li, and P. Willis, Adv. Mater., 15, 1161 (2003).

19. M. Molina-Sabio, M. T. Gonzalez, F. Rodriguez-Reinoso, and A. Sepulveda-Escribano, Carbon, 34, 505 (1996).

20. A. Addoun, J. Dentzer, and P. Ehrburger, Carbon, 40, 1140 (2002).

21. T. Liu, T. V. Sreekumar, S. Kumar, R. H. Hauge, and R. E. Smalley, Carbon, 41, 2427 (2003).

22. B. Wang, X. Y. Liu, H. M. Liu, D. X. Wu, H. P. Wang, J. M. Jiang, X. B. Wang, P. A. Hu, Y. Q. Liu, and D. B. Zhu, J. Mater. Chem., 13, 1124 (2003).

23. X. B. Wang, Y. Q. Liu, and D. B. Zhu, Adv. Mater., 14, 165 (2002).

24. M. S. P. Shaffer, X. Fan, and A. H. Windle, Carbon, 36, 1603 (1998).

25. R. Bandyopadhyaya, E. Nativ-Roth, O. Regev, and R. Yerushalmi-Rozen, Nano Lett., 2, 25 (2002).

26. B. Wang, J. M. Li, H. P. Wang, J. M. Jiang, and Y. Q. Liu, Macromol. Symp., 116, 189 (2004).

27. T. V. Sreekumar, T. Liu, B. G. Min, H. Guo, S. Kumar, R. H. Hauge, and R. E. Smalley, Adv. Mater., 16, 58 (2004).

28. J. C. Grunlan, W. W. Gerberich, and L. F. Francis, J. Appl. Polym. Sci., 80, 892 (2001). 